\title{
Cardiac Marker Troponin in Dubai Marathon Runners
}

\author{
Ghulam Naroo, Zulfiqar Ali, Mohamed Hanif Shaji, Behrooz Hussain Vazirian, Mousmeh Baradaran Falahkhir \\ and Moin Fikree \\ Emergency Department, Rashid Hospital Trauma Center, Dubai 4545, UAE
}

\begin{abstract}
Professional marathoners demonstrate abnormal rises in cardiac biomarkers and a spectrum of alterations in a 12-lead ECG (electrocardiogram). Such alterations are likely the consequence of athletic conditioning and represent another potential component of the athletic heart syndrome. It remains unclear, however, whether the exercise induced change in ECG or the increase in cardiac biomarkers in obviously healthy athletes is of any consequence. Abnormal levels of cardiac biomarkers in marathon runners may inevitably lead to a misdiagnosis. One hundred healthy marathon runners were enrolled in this study during Dubai Marathon, Jan. 2010. ECGs were conducted and blood samples were collected 30 min before and less than 30 minutes after the race. A baseline renal function was obtained on these samples and a level of cTnT (cardiac Troponin T) was measured. 13\% (7/52) exhibited ECG abnormalities before and 17\% (9/52) after the race. There were no abnormal troponin levels before the race but levels increased by an average of $14.28 \%$ after the race. This was not related to changes on the ECG. Only one participant with an abnormal troponin level also had an abnormal pattern on ECG. Diagnostic biochemical tests are prone to changes following exercise. An increase in the cardiac biomarker Troponin T may be transient following marathon run. No correlation between the ECG pattern and a raised Troponin $\mathrm{T}$ has been found.
\end{abstract}

Key words: Cytotoxicity, crude extracts, capparis spinosa, fruit, tumor cell lines.

\section{Introduction}

It is a well documented fact that physical activity provides many benefits to cardiovascular health such as lowering blood pressure, improving lipid profile, modulating insulin resistance and decreasing overall mortality [1]. However, the occurrence of cardiac death and other adverse events post marathon run has also been well documented in the medical literature of Ref. [2-5]. The data on the impact of screening programs is fairly limited and no randomized trial has ever been published in that direction. This may possibly reflect an extremely low incidence of sudden cardiac death in athletes, still it cannot entirely be ruled out that the possibility of preventing and perhaps even identifying athletes at risk of such adverse outcomes may very well exist.

Recent studies [6-11] have demonstrated the risks of exercise-associated cardiac damage and/or dysfunction, as evidenced by a rise in cardiac

Corresponding author: Ghulam Naroo, FRCP, research field: emergency medicine. E-mail: gynaroo@dha.gov.ae. biomarkers. Of particular interest is the transient rise in activities of creatine kinase MB [12] following marathon run. Despite the evidence, there continues to be considerable debate over the consequence of such elevations in cardio specific markers such as troponin $[13,14]$. That exercise can induce increases in cTnT (cardiac Troponin T) or cTnI (cardiac Troponin I) in obviously healthy athletes has been demonstrated in several studies in the past and has been the subject of a recent meta analysis [9] which concluded that nearly half the endurance athletes that have been studied have demonstrated a rise in $\mathrm{cTnT}$.

The athletic heart syndrome is a well described phenomenon in the medical literature of Ref. [15]. It is known to occur in trained athletes and is characterized by an enlargement of the heart muscle due to significant amounts of exercise. Studies have documented a variety of electrocardiographic changes in trained athletes, some of which include increased precordial $\mathrm{R}$-wave or $\mathrm{S}$-wave voltages, $\mathrm{T}$-wave inversion and deep Q-wave changes. Although this 
does raise the possibility of a pathological heart condition, it has more often been attributed to the cardiac morphological remodeling induced by the athletic condition [16-20]. Furthermore, the heart of an athlete may also demonstrate sinus bradycardia, right atrial enlargement, conduction delays like 1st and possibly 2 nd degree heart block as well as early repolarization $[21,22]$.

Although the need to further study such claims is recognized, the authors of this paper aim at studying the hypothesis that a rise in cTnT is related to myocardial damage in otherwise healthy athletes. We have, therefore, attempted to identify the relationship between cTnT and ECG in a group of athletes subjected to the vigorous exertion of a marathon.

\section{Methods}

One hundred participants were randomly enrolled in this study. Of the 400 marathon runners registered to run the $42 \mathrm{~km}$ race at the annual Dubai Marathon, we chose the first 100 even numbers. The cosmopolitan nature of the city allowed us to recruit runners of 22 nationalities (including most of Western Europe, northern and central Africa and the Middle East). All runners provided written consent and completed a comprehensive screening form prior to the race. This was in the form of a questionnaire that included questions about current and past medical conditions, family history and the use of prescription medications, if any.

Blood samples were collected $30 \mathrm{~min}$ before and less than $30 \mathrm{~min}$ after the race. Samples were then sent to the Rashid Hospital Trauma Center laboratory and measured using electrochemiluminescence. Urea and creatinine were also measured on all samples for the purpose of obtaining a baseline renal function. ECGs were also performed on all participants before and less than 30 min after the race.

Of the 100 enrolled participants, only 52 completed the study and underwent a post race electrocardiography. Of these 52, only 21 participants gave blood samples for a post race Troponin $\mathrm{T}$ level. Only men between the ages of 25 and 45 were included in this study. We felt including age among the variables was warranted since according to the American Heart Association guidelines abnormal ECG patterns in athletes below the age of 35 may be indicative of a congenital heart disease [23]. Of the 52 participants that did complete the study with follow up electrocardiograms, 20 were under the age of 35 and 32 were above the age of 35 . Individuals with history of diabetes, hypertension or coronary artery disease were excluded. Also individuals with a positive family history of sudden death were not enrolled. The runners were divided into two groups. Regular runners $(n=46)$ were defined as those with 12 weeks of preparation, 5 times a week with the longest run lasting $2 \mathrm{~h}$ and aiming to run 20-40 m per week. Beginners $(n=6)$ were those under the "get you round" program who had had no formal training.

ECGs performed on all participants were independently interpreted by three trained internal physicians and one trained emergency physician.

\section{Results}

13\% (7/52) exhibited ECG abnormalities before the race (Fig. 1). These included $\mathrm{T}$-wave inversions, incomplete Right Bundle Branch Block, early repolarization and $\mathrm{LVH}$ pattern. Post race this number rose to seventeen percent (9/52) (Fig. 2). Supraventricular tachycardias (SVT) were seen on both of these abnormal ECGs, although both were transient and neither required medical treatment. The pre-race ECGs of both these candidates showed normal sinus rhythm, no conduction defect or ST segment abnormality. Moreover, both these participants were regular runners.

The cutoff value for Troponin $\mathrm{T}$ at our institution is $\leq 0.02 \mathrm{ng} / \mathrm{mL}$. There was no raised troponin identified on any of the samples sent before the race. After the race, however, troponin levels increased by an 

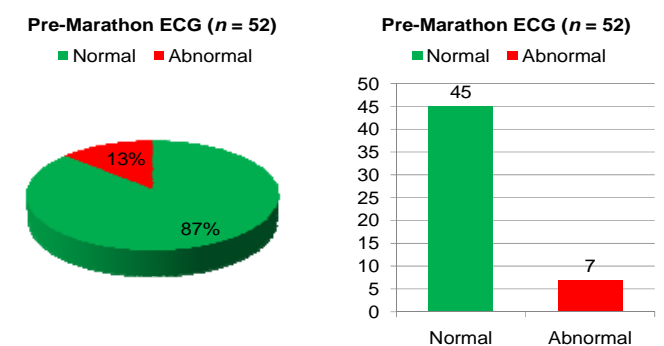

Fig. 1 This graph shows the number of participants with abnormal ECGs prior to the race.
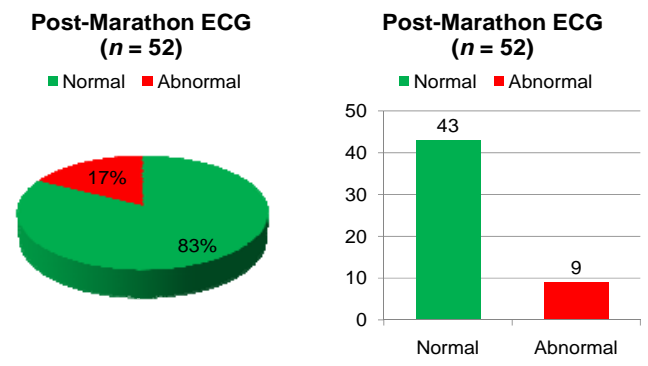

Fig. 2 This graph shows the number of participants with abnormal ECGs less than 30 min after the race.

average of $14.28 \%$ (Fig. 3) but this was not associated with any ECG changes. These levels fell back to normal $8 \mathrm{~h}$ post exercise.

The relationship between the raised troponin and ECG patterns is illustrated in Fig. 4. Troponin T (TnT) was found positive in two runners with a normal ECG and one runner with an abnormal ECG (Fig. 5 and Table 1).

\section{Discussion}

Endurance exercise can induce increases in cardiac troponin in healthy endurance athletes under special conditions (especially during endurance competitions), which do not represent irreversible myocardial damage but probably reversible transitory membrane leakage of cardiomyocytes and, therefore, seem to be without pathological significance. The exercise-induced releases of free cytoplasmic cardiac

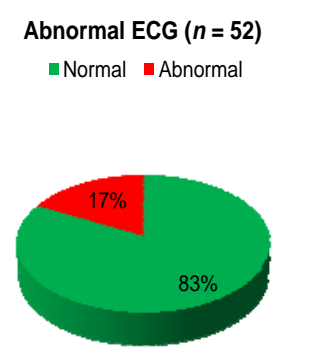

Elevated TnT $(n=21)$

- Normal -Elevated TnT

Fig. 3 This graph shows the number of participants with a raised TnT less than 30 min after the race. Abnormal ECGs $(9 / 52)=17.3 \%$, Elevated TnT $(3 / 21)=14.3 \%$

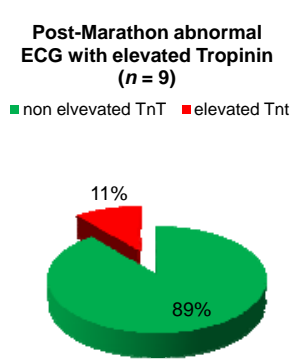

Elevated TnT $(n=21)$ - Normal - Elevated TnT

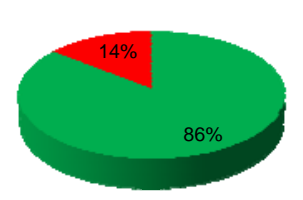

Fig. 4 This graph illustrates the relationship between positive Troponin $T$ values and post-marathon ECGs. TnT was found positive in two runners with normal ECG patterns and one with an abnormal ECG pattern. Normal ECGs with Elevated troponin $=4.65 \%(2 / 43)$, Abnormal ECGs with Elevated troponin $=11.1 \%(1 / 9)$.

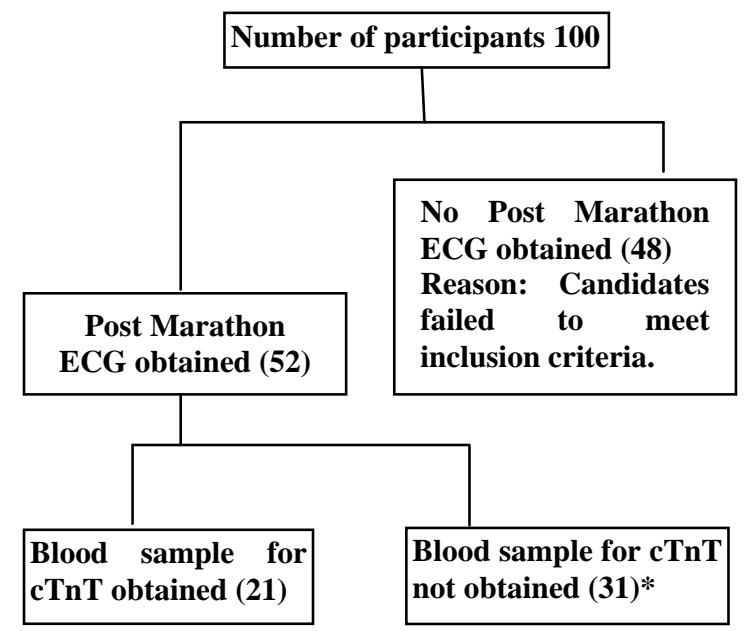

* Candidates did not give follow up blood samples.

Fig. 5 Flowchart illustrating the number of participants ECGs and blood samples were obtained on.

Table 1 Details of participants with an abnormally high TnT.

\begin{tabular}{clllllll}
\hline Case No. & Age & Level of training & Relevant history & Pre-race ECG & Post-race ECG & Pre-race TnT & Post-race TnT \\
\hline 152 & 41 & Regular & None & Normal & Normal & Negative & 0.023 \\
44 & 39 & Regular & None & Normal & Normal & Negative & 0.039 \\
118 & 38 & Regular & None & LVH & LVH & Negative & 0.021 \\
\hline
\end{tabular}


troponins as well as B-type natriuretic peptide may induce cardiomyocytes' adaptation on endurance exercise and modulate myocardial hypertrophy in otherwise healthy athletes. The underlying cellular mechanisms of exercise induced releases in cardiac troponins and (N-terminal prohormone brain natriuretic peptide) B-type natriuretic peptide, however, have to be studied in the future to better understand their role in endurance exercise on the athletic heart [13, 24, 25].

The mechanisms responsible for post-exercise cTnT release are not known. Some have suggested that exercise-induced cardiac troponin release precedes physiologic cardiac hypertrophy $[6,8,26,27]$. Release of acidic and basic fibroblast growth factor $(>$ FGF and AFGF, respectively) from the cytosol of cardiac myocytes has been demonstrated, occurring in response to membrane damage caused by an increase in the rate and force of cardiac contraction. Membrane damage, subsequent to an increased rate and force of cardiac contraction during endurance exercise, may prove a mechanism by which cytosolic troponin is released into the circulation. High-intensity exercise, defined as high intensity running for $30 \mathrm{~min}$, is also known to result in cTnI release [28].

Although moderate aerobic exercise has documented health benefits [1], the long term cardiac implications of repeated release of cTnT after multiple endurance events are less clear. Whether post-exercise cTnT release is related to micro-injury of the myocardium is presently not clear. Cross-sectional and longitudinal studies are required to further examine the causes and consequences of post-exercise cTnT release.

The limitations of this study include a small sample size. Also, troponin levels at $24 \mathrm{~h}$ were not performed but that is only because all abnormal troponin levels had returned to normal within $8 \mathrm{~h}$. A creatinine clearance was substituted with a one time reading of urea and creatinine; therefore, it lacked a proper assessment of renal function. Finishing time of participants were not recorded.

There is no financial support or conflict of interest.

\section{Conclusions}

In conclusion, many of the diagnostic biochemical tests on which we rely are affected by exercise. Physicians needs to be aware of these effects when interpreting results in clinical setting, so a full history is essential before interpreting any abnormality in these indices in order to avoid over-diagnosing marathon runners in particular with any pathology.

In contrast to acute myocardial infarction, increase in cTnT is only mild and of shorter duration in healthy athletes. Exercise-associated elevations in cTnT decreased well before the $8 \mathrm{~h}$ mark so it may safely be concluded that values reach normal limits within such a time frame.

Our study shows no correlation between the ECG pattern and a raised troponin. Blood tests in marathon runners may produce a raised troponin post exercise. Screening of the athletic population of cardiovascular risk by doing 12 lead ECGs is not a proven diagnostic tool.

\section{References}

[1] R.S. Paffenbarger, R.T. Hyde, A.L. Wing, I. Lee, D.L. Jung, J. B. Kampert, The association of changes in physical-activity level and other lifestyle characteristics with mortality among men, N. Engl. J. Med. 328 (8) (1993) 538-545.

[2] M.A. Mittleman, M. Maclure, G.H. Tofler, J.B. Sherwood, R.J. Goldberg, J.E. Muller, Triggering of acute myocardial infarction by heavy physical exertion-Protection against triggering by regular exertion, N. Engl. J. Med. 329 (23) (1993) 1677-1683.

[3] B.J. Maron, L.C. Poliac, W.O. Roberts, Risk for sudden cardiac death associated with marathon running, J. Am. Coll. Cardiol. 28 (2) (1996) 428-431.

[4] N.B. Ratliff, K.M. Harris, S.A. Smith, M. Tankh-Johnson, C.C. Gornick, B.J. Maron, Cardiac arrest in a young marathon runner, Lancet 360 (9332) (2002) 542.

[5] P.D. Thompson, The cardiovascular complications of vigorous physical activity, Arch. Intern. Med. 156 (20) (1996) 2297-2302.

[6] A. Koller, Exercise-induced increases in cardiac 
troponins and prothrombotic markers, Med. Sci. Sports Exerc. 35 (3) (2003) 444-448.

[7] J. Scharhag, R. Shave, K. George, G. Whyte, W. Kindermann, Exercise-induced increases in cardiac troponins in endurance athletes: A matter of exercise duration and intensity?, Clin. Res. Cardiol. 97 (1) (2008) 62-63.

[8] J. Scharhag, A. Urhausen, G. Schneider, M. Herrmann, K. Schumacher, M. Haschke, et al., Reproducibility and clinical significance of exercise-induced increases in cardiac troponins and $\mathrm{N}$-terminal pro brain natriuretic peptide in endurance athletes, Eur. J. Cardiovasc. Prev. Rehabil. 13 (3) (2006) 388-397.

[9] R. Shave, K.P. George, G. Atkinson, E. Hart, N. Middleton, G. Whyte, et al., Exercise-induced cardiac troponin T release: A meta-analysis, Med. Sci. Sports Exerc. 39 (12) (2007) 2099-2106.

[10] R. Shave, K. George, D. Gaze, The influence of exercise upon cardiac biomarkers: A practical guide for clinicians and scientists, Curr. Med. Chem. 14 (13) (2007) 1427-1436.

[11] G. Whyte, N. Stephens, R. Senior, K. George, R. Shave, M. Wilson, et al., Treat the patient not the blood test: The implications of an increase in cardiac troponin after prolonged endurance exercise, British Journal of Sports Medicine 41 (9) (2007) 613-615.

[12] E.M. Ohman, K.K. Teo, A.H. Johnson, P.B. Collins, D.G. Dowsett, J.T. Ennis, et al., Abnormal cardiac enzyme responses after strenuous exercise: Alternative diagnostic aids, Br. Med. J. (Clin. Res. Ed) 285 (6354) (1982) 1523-1526.

[13] A.J. Siegel, E.L. Lewandrowski, K.Y. Chun, M.B. Sholar, A.J. Fischman, K.B. Lewandrowski, Changes in cardiac markers including B-natriuretic peptide in runners after the Boston marathon, Am. J. Cardiol. 88 (8) (2001) 920-923.

[14] A.J. Siegel, M. Sholar, J. Yang, E. Dhanak, K.B. Lewandrowski, Elevated serum cardiac markers in asymptomatic marathon runners after competition: Is the myocardium stunned?, Cardiology 88 (6) (1997) 487-491.

[15] J.T. Lohr, Athletic Heart Syndrome, 3rd ed., in: L. Jacqueline (Eds.), Longe, 2006.

[16] A. Venerando, V. Rulli, Frequency morphology and meaning of the electrocardiographic anomalies found in olympic marathon runners and walkers, J. Sports Med. Phys. Fitness 50 (1964)135-141.
[17] N. Hanne-Paparo, M.H. Wendkos, D. Brunner, T wave abnormalities in the electrocardiograms of top-ranking athletes without demonstrable organic heart disease, Am. Heart J. 81 (6) (1971) 743-747.

[18] J. Lichtman, R.A. O'Rourke, A. Klein, J.S. Karliner, Electrocardiogram of the athlete: Alterations simulating those of organic heart disease, Arch. Intern. Med. 132 (5) (1973) 763-770.

[19] B. J. Maron, A. Pelliccia, The heart of trained athletes: Cardiac remodeling and the risks of sports, including sudden death, Circulation 114 (15) (2006) 1633-1644.

[20] T.P. Huston, J.C. Puffer, W.M. Rodney, The athletic heart syndrome, N. Engl. J. Med. 313 (1) (1985) 24-32.

[21] P.D. Thompson, Cardiovascular adaptations to marathon running: The marathoner's heart, Sports Med. 37 (4-5) (2007) 444-447.

[22] P.D.S. Tunstall, Marathon cardiac deaths: The london experience, Sports Med. 37(4-5) (2007) 448-450.

[23] B.J. Maron, P.D. Thompson, J.C. Puffer, C.A. McGrew, W.B. Strong, P.S. Douglas, et al., Cardiovascular preparticipation screening of competitive athletes: Addendum - An addendum to a statement for health professionals from the Sudden Death Committee (Council on Clinical Cardiology) and the Congenital Cardiac Defects Committee (Council on Cardiovascular Disease in the Young), American Heart Association, Circulation 97 (22) (1998) 2294.

[24] A. Kratz, K.B. Lewandrowski, A.J. Siegel, K.Y. Chun, J.G. Flood, E.M. Van Cott, et al., Effect of marathon running on hematologic and biochemical laboratory parameters, including cardiac markers, Am. J. Clin. Pathol. 118 (6) (2002) 856-863.

[25] A.J. Siegel, J.J. Stec, I. Lipinska, E.M. Van Cott, K.B. Lewandrowski, P.M. Ridker, et al., Effect of marathon running on inflammatory and hemostatic markers, Am. J. Cardiol. 88 (8) (2001) 918-20, A9.

[26] A. Koller, Postexercise increases in cardiac troponin T and brain natriuretic peptide, Med. Sci. Sports Exerc. 36 (4) (2004) 736.

[27] R. Shave, E. Dawson, G. Whyte, K. George, M. Nimmo, J. Layden et al., The impact of prolonged exercise in a cold environment upon cardiac function, Med. Sci. Sports Exerc. 36 (9) (2004) 1522-1527.

[28] R. Shave, P. Ross, D. Low, K. George, D. Gaze, Cardiac troponin $\mathrm{I}$ is released following high-intensity short-duration exercise in healthy humans, Int. J. Cardiol. 145 (2) (2010) 337-339. 